\title{
Neonatal Death
}

National Cancer Institute

\section{Source}

National Cancer Institute. Neonatal Death. NCI Thesaurus. Code C80082.

Death of a live newborn during the first 28 days of life. 